\title{
A STUDY OF OBESITY AS A SOLE CAUSE OF DYSLIPIDAEMIA IN YOUNG ADULTS IN A RURAL SETUP
}

\author{
Amandeep Singh Kaloti ${ }^{1}$, Anoop Chhabra², E. Chandrasekaran ${ }^{3}$ \\ ${ }^{1}$ Associate Professor, Department of Medicine, S. G. T. Medical College, Budhera, Gurgaon. \\ ${ }^{2}$ Associate Professor, Department of Medicine, S. G. T. Medical College, Budhera, Gurgaon. \\ ${ }^{3}$ Professor, Department of Medicine, S. G. T. Medical College, Budhera, Gurgaon.
}

\begin{abstract}
Obesity is widely prevalent now-a-days. The prevalence is only going to increase with time. This has a deleterious effect on the body in the form that various diseases associated with obesity occur in the affected individuals. One of this is dyslipidaemia.

AIM

This cross sectional study was done to know the occurrence of obesity as a sole cause for dyslipidaemia in young adults in a rural setup.

MATERIALS AND METHODS

In this study, 610 patients were studied in a one-year period. Those patients with a BMI of $>30$ were included in the study. BMI was calculated, waist circumference was noted and findings of lipid profile were gathered. Obese patients with previous medical problems like diabetes mellitus, hypertension, thyroid disease, heart disease, etc. were excluded from the study. Obesity as a sole cause for dyslipidaemia was looked for in the patients by doing fasting lipid profile in the patients.
\end{abstract}

\section{RESULTS}

We found that in obese patients with a BMI of $>30$, the waist circumference was high and these patients had derangements in the lipid profile where the TG were high in 407 (66.72\%) patients, HDL was low in 416 (68.20\%) patients and LDL was high in 423 (69.34\%) patients. So, these patients of obesity had derangements in the fasting lipid profile.

\section{CONCLUSION}

Obesity is an important cause of dyslipidaemia. Lipid levels should be investigated in all the young obese patients as high lipid levels in these patients leads to various cardiovascular complications. Therefore, by checking the lipid profile in obese patients, we can prevent the cardiovascular complications from occurring in these patients.

\section{KEYWORDS}

Obesity, Dyslipidaemia, Young Adults, BMI, Lipid Profile.

HOW TO CITE THIS ARTICLE: Kaloti AS, Chhabra A, Chandrasekaran E. A study of obesity as a sole cause of dyslipidaemia in young adults in a rural setup. J. Evolution Med. Dent. Sci. 2016;5(39):2401-2404, DOI: 10.14260/jemds/2016/558

\section{INTRODUCTION}

Young adults in India are having a high prevalence of overweight and obesity, which is increasing rapidly in the developing countries. The current trends will accelerate over time.1,2,3 Obesity is associated with multiple health problems including dyslipidaemia apart from the other health problems. ${ }^{3}$ It is important for the physicians to identify, evaluate and treat patients for obesity and associated comorbid conditions like dyslipidaemia.1,2,3

In the presence of nutritional abundance and sedentary lifestyle and influences importantly by genetic endowment, the body's system increases the adipose energy stores and produces adverse health consequences. Obesity is a state of excess adipose tissue mass. Obesity is more effectively defined by assessing its linkage to the morbidity and mortality. $4,5,6$

Large scale epidemiological studies suggest that allcause metabolic, cancer and cardiovascular morbidity begin

Financial or Other, Competing Interest: None.

Submission 30-03-2016, Peer Review 22-04-2016,

Acceptance 30-04-2016, Published 16-05-2016.

Corresponding Author:

Dr. Amandeep Singh Kaloti,

H. No. 1231, Urban Estate,

Phase 1, Jalandhar-144022,

Punjab.

E-mail: amansaini1231@rediffmail.com

DOI: $10.14260 /$ jemds $/ 2016 / 558$ to rise at BMI $>25$. BMI between 25 and 30 should be viewed as medically significant and worthy of therapeutic intervention. 6

The distribution of adipose tissue in different anatomic deposits also has substantial implications for morbidity. Specifically, intra-abdominal and abdominal subcutaneous fat have more significance than subcutaneous fat in the buttocks and lower extremities. Many of the most important complications of obesity such as insulin resistance, diabetes, hypertension, hyperlipidaemia, hyperandrogenism in women are linked more strongly to intra-abdominal and/or upper body fat than to overall obesity. This may relate to the fact that intra-abdominal adipocytes are more lipolytically active than those from other deposits. Release of free from acids into the portal circulation has adverse metabolic actions, especially on the liver. ${ }^{6}$

Adipocyte is also an endocrine cell that releases numerous molecules in regulated fashion. These include energy balance regulating hormone leptin, cytokines such as TNF and IL-6, complement factors such as factor D, prothrombotic agents such as PAI-1, and angiotensinogen. These factors and those not yet identified play a role in physiology of lipid homeostasis, insulin sensitivity, blood pressure control, coagulation and vascular health and are likely to contribute to obesity related pathologies. Various genetic forms of obesity have been defined with Mendelian inheritance patterns. Body weight 'set point' idea is 
supported by physiological mechanisms centered around sensing systems in adipose tissue that reflects fat stores and a receptor or "adipostat" that is in the hypothalamic centres. Disruption of this adipostat signalling leads to obesity. ${ }^{6}$ Obesity is associated with an increase in mortality with a 50 $100 \%$ increased risk of death from all causes compared to normal weight individuals.

Three key anthropometric measurements are important in evaluating the degree of obesity: weight, height and waist circumference. The Body Mass Index (BMI) calculated as weight $(\mathrm{Kg}) /$ height $(\mathrm{mt})^{2}$ is used to classify the weight status and the risk of disease. These parameters help to measure and quantitate the obesity. All have different defined cut-off upper limits for measuring the obesity. This helps to label the obese patients into different categories and severities of obesity. It also helps when one plans for interventions in obese patients as higher category of obesity will require more drastic interventions.

\begin{tabular}{|c|c|c|c|}
\hline $\begin{array}{c}\text { Disease } \\
\text { Risk }\end{array}$ & Classification & $\begin{array}{c}\text { BMI } \\
(\mathbf{K g} / \mathbf{m} 2)\end{array}$ & $\begin{array}{c}\text { Obesity } \\
\text { Class }\end{array}$ \\
\hline Low & Underweight & $<18.5$ & - \\
\hline Low & Healthy Weight & $18.5-24.9$ & - \\
\hline Increased & Overweight & $25-29.9$ & - \\
\hline High & Obesity & $30-34.9$ & I \\
\hline Very High & Obesity & $35-39.9$ & II \\
\hline $\begin{array}{c}\text { Extremely } \\
\text { High }\end{array}$ & $\begin{array}{c}\text { Extreme } \\
\text { Obesity }\end{array}$ & $\geq 40$ & III \\
\hline
\end{tabular}

Excess abdominal fat, assessed by measurement of waist circumference is associated with higher risk of comorbid conditions. Measurement should be performed in the horizontal plane above the iliac crest.

Cut-point Values for Waist Circumference in South Asians:

Men - 37 inches.

Women - 31.5 inches.

For all patients, a fasting lipid profile should be performed including Total Cholesterol (TC), Low Density Lipoprotein (LDL), High Density Lipoprotein (HDL), Triglyceride (TG) levels.7,8,9,10

Lipoproteins are large macromolecular complexes that play an essential role in the absorption of dietary cholesterol, long chain fatty acids and fat soluble vitamins, transport of triglycerides, cholesterol and fat soluble vitamins from the liver to peripheral tissues; and the transport of cholesterol from peripheral tissues to the liver. The plasma lipoproteins are divided into 5 major classes based on their relative density: chylomicrons, VLDL, IDL, HDL, LDL. ${ }^{11}$

Obesity is frequently accompanied by dyslipidaemia. The increase in the adipocyte mass and accompanying decreased insulin sensitivity associated with obesity has multiple effects on lipid metabolism. More free fatty acids are derived from the expanded adipose tissue to the liver, where they are re-esterified in hepatocyte to form triglycerides, which are packaged into VLDLs for secretion into the circulation. The increased insulin levels promote fatty acid synthesis in the liver. Increased dietary intake of simple carbohydrates also drives hepatic production of VLDL, resulting in elevation in VLDL and/or LDL in some obese subjects. Plasma levels of HDL tend to be low in obesity, due in part to the lipolysis. ${ }^{11}$

Guidelines for screening and management of lipid disorders has been provided by an expert Adult Treatment Panel (ATP) convened by the National Cholesterol Education Program (NCEP) of the National Heart, Lung and Blood Institute. The NCEP ATP III guidelines published in 2001 recommend that all adults over age of 20 years should have plasma levels of cholesterol, triglycerides, LDL, HDL measured after a 12-hour overnight fast. In most clinical laboratories, the total cholesterol and triglycerides in the plasma are measured enzymatically and then the cholesterol in the supernatant is measured after precipitation of apo-B containing lipoproteins to determine the HDL. The LDL level is estimated by using the following equation.

LDL=Total cholesterol-(TG/5)-HDL

This formula is reasonably accurate if the test is obtained in a fasting state. Multiple epidemiological studies have shown the association of dyslipidaemia with CVD. The dyslipidaemia due to obesity shows increased TG, decreased HDL and increased LDL. ${ }^{11}$

\section{AIMS AND OBJECTIVES}

To study the occurrence of obesity as sole cause of dyslipidaemia in young adults in a rural setup.

\section{Inclusion Criteria}

All young patients attending the medicine OPD and admitted in the medicine wards who were obese and were between 18-30 years of age were included in the study, i.e. those with BMI 30 or more.

\section{Exclusion Criteria}

All the young patients with previous medical problems like diabetes mellitus, hypertension, thyroid disorders, kidney disease, liver disease, heart disease were excluded.

\section{MATERIALS AND METHODS}

This was a cross sectional study done at SGT Medical College and Research Institute, Budhera, Gurgaon, in the Department of Medicine. The study period was from 1st July 2013 to the 30th June 2014. Approval from the Ethics Committee was taken. Patients admitted to the wards and attending the OPD were investigated. Patients were explained in detail about the study and the purpose of the study. Detailed written consent regarding the willingness to participate in the study was taken from the patients.

Data of the patients was collected from the patient records of the patients who attended the OPD or were admitted in the ward of the Medicine Department. Demographic details and pre-existing medical problems were required and those found to have previous medical problems were excluded from the study.

Data regarding the BMI, waist circumference and fasting lipid profile were gathered.

Data regarding the fasting blood sugar, blood pressure, thyroid function test, liver function tests and renal function test were also gathered for the exclusion criteria. Patient having derangements in any of these were excluded from the study.

Patients were subjected to measurements of height, which was recorded. Weight was recorded with the weighing 
machine available in the wards of the medicine and OPD. Same weighing machine was used for all the patients. Waist circumference was carefully measured in each patient and recorded.

610 obese patients were studied in the one year period. Those patients with a BMI of 30 or more were studied. During this period, dyslipidaemia was looked for in them.

\begin{tabular}{|c|c|c|}
\hline Age Group (Years) & No. of Cases & \% \\
\hline $18-20$ & 130 & 21.31 \\
\hline $21-25$ & 180 & 29.51 \\
\hline $26-30$ & 300 & 49.18 \\
\hline \multicolumn{2}{|c|}{ Table I: Table showing the Demographic } \\
Details of the Patients \\
\hline
\end{tabular}

\begin{tabular}{|c|c|c|}
\hline BMI (kg/ $\mathbf{m}^{\mathbf{2}}$ ) & No. of Cases & \% \\
\hline $30-34.9$ & 157 & 25.74 \\
\hline $35-39.9$ & 310 & 50.82 \\
\hline$\geq 40$ & 143 & 23.44 \\
\hline \multicolumn{2}{|c|}{ Table II: Table showing the BMI of the } \\
Patients in the Study \\
\hline
\end{tabular}

\begin{tabular}{|c|c|c|}
\hline Gender & No. of Cases & \% \\
\hline Male & 351 & 57.54 \\
\hline Female & 259 & 42.46 \\
\hline \multicolumn{2}{|c|}{ Table III: Table showing the Gender } \\
of the Patients in the Study \\
\hline
\end{tabular}

\begin{tabular}{|c|c|c|}
\hline Waist Circumference & No. of Cases & \% \\
\hline Male $>37$ inches & 351 & 57.54 \\
\hline Women >31.5 & 259 & 42.46 \\
\hline \multicolumn{2}{|c|}{ Table IV: Table showing the Waist } \\
Circumference in the Patients \\
\hline
\end{tabular}

\begin{tabular}{|c|c|c|}
\hline $\begin{array}{c}\text { Lipid Parameter } \\
\text { Total Cholesterol (TC) }\end{array}$ & $\begin{array}{c}\text { No. of Cases } \\
\mathbf{3 1 8}\end{array}$ & $\mathbf{9 2 . 1 3}$ \\
\hline LDL & 423 & 69.34 \\
\hline HDL & 416 & 68.20 \\
\hline TG & 407 & 66.72 \\
\hline \multicolumn{2}{|c|}{$\begin{array}{c}\text { Table V: Table showing the Abnormality } \\
\text { in Lipid Profile of the Patients }\end{array}$} \\
\hline
\end{tabular}

\section{DISCUSSION}

Most of the patients in the study were in the age group of 2630 yrs., in which these were 300 patients (i.e. 49.18\%). The next large group was those between 21-25 yrs. of age in which there were 180 patients (i.e. 29.51\%), whereas between 18-20 yrs. of age there were 130 patients (i.e. $21.3 \%$ ). This finding indicates that the obesity increases with age and obese patients with a BMI $>30$ are in the latter part of the young age. 12,13

157 patients i.e. $25.74 \%$ of the patients were in the obesity class I, 310 patients (50.82\%) were in the obesity class II and 143 patients (23.44\%) were in the obesity class III. This indicates that those who are obese are having the BMI in the high range. This helps us to decide and recommend treatment modalities for those obese. These are the patients who are prone for various comorbidities of obesity. ${ }^{14}$

Out of all the cases, 351 patients $(57.54 \%)$ were males whereas 259 (42.46\%) were females. This shows that males and females are both prone for obesity and its complications.
All the male and female patients met the criterion of waist circumference of being $>37$ inches in males and $>31.5$ inches in females. This shows that the patients who are obese also have a high waist circumference. These set defined parameters allow us to segregate the obese patients from the others.

Lipid parameters showed that there was a rise in the total cholesterol in 318 (52.13\%) patients. LDL was high in $423(69.34 \%)$ patients. HDL cholesterol was low in 416 $(68.20 \%)$ of patients and TG were high in 407 (66.72\%) of the patients. These findings indicate that most of the young adults who are obese also have derangement in the lipid profile. ${ }^{12,13}$

Lipid parameter derangement showed that the TG were increased in $66.72 \%$ of patients. HDL was decreased in $68.20 \%$ of patients and LDL was increased in $69.34 \%$ of patients, which shows that these parameters were deranged in a large number of obese patients. This may be due to an increase in the adipocyte mass leading to decreased insulin sensitivity. This was as in the literature. ${ }^{13}$

So, a large number of obese patients have a derangement in their lipid profile with obesity as a sole cause. Most of these young adults have class II obesity and so are having a very high risk of developing comorbidities.

\section{CONCLUSION}

Obesity is an important cause of dyslipidaemia. Lipid levels should be investigated in young obese persons. This helps to advice proper life-style changes and drug treatment to those having an abnormality in the lipid levels and so can help prevent the complications of dyslipidaemia from occurring. This can be an important intervention in those who are obese and can act as a red flag or a warning signal to obese patients, asking them to reduce weight and also to get the timely treatment for dyslipidaemia.

\section{REFERENCES}

1. Daphne P Guh, Wei Zhang, Nick Bansback, et al. Incidence of comorbidities related to obesity and overweight: a systematic review and meta-analysis. BMC Public Health BMC series 2009;9:88.

2. Elena Martin-Rodriguez, Francisco Guillen-Grima, Amelia Marti, et al. Comorbidity associated with obesity in a large population: the APNA study. Obesity Research and Clinical Practice 2015;9(5):435-47.

3. Jia WP, Xiang KS, Chen L, et al. Epidemiological study on obesity and its comorbidities in Urban Chinese older than 20 years of age in Shangai, China. Obesity Review 2002;3(3):157-65.

4. Neel JV. Diabetes mellitus: a 'thirfty' genotype rendered detrimental by 'progress'? American Journal of Human Genetics 1962;14(4):353-62.

5. Selby JV, Newman B, Quesenberry CP, et al. Evidence of genetic influence on central body fat in middle-aged twins. Hum Biol 1989;61(2):179-94.

6. Adams KF, Schatzkin A, Harris TB, et al. Overweight, obesity and mortality in a large prospective cohort of persons 50-71 years old. New England Journal of Medicine 2006;355(8):763-78.

7. Singh AK, Singh SK, Singh N, et al. Obesity \& dyslipidaemia. Int J Biol Med Res 2011;2(3):824-8. 
8. Howard BV, Ruotolo G, Robbins DC. Obesity and dyslipidaemia. Endocrionoal Metab Clin North Am 2003;32(4):855-67.

9. Boudewijn Klop, Jan Willem F Elte, Manuel Castro Cabezas. Dyslipidaemia in obesity: mechanisms and potential targets. Nutrients 2013;5(4):1218-40.

10. Vaneeta Bamba, Daniel J Rader. Obesity and atherogenic dyslipidaemia-gastroenterology.

www.gastrojournal.org/article/S0016-5085 (07) 005823 /abstract.

11. Council on Scientific Affairs, Division of Scientific Analysis and Technology, American Medical Association. Dietary and pharmacologic therapy for lipid risk factors. JAMA 1983;250:1873-9.
12. India facing obesity epidemic: experts: The Hindu 2007;10-12.

13. National Family Health Survey, 2005-06. Mumbai: International Institute for population Sciences 2007; Vol 1.

14. Misra A, Pandey RM, Devi JR, et al. Corrigendum to high prevalence of diabetes, obesity and dyslipidaemia in urban slum population in India. Inter J of Obesity Related Metabolic Disorders 2001;25(11):1722-9. 